\title{
Core-shell nanocomposite of flower-like molybdenum disulfide nanospheres and molecularly imprinted polymers for electrochemical detection of anti COVID-19 drug favipiravir in biological samples
}

\author{
Shuang Wang ${ }^{1} \cdot$ Chen Wang ${ }^{1} \cdot$ Yuxiao $\mathrm{Xin}^{1} \cdot{\text { Qiuyun } \mathrm{Li}^{1} \cdot \text { Weilu Liu }}^{1}$ (])
}

Received: 23 November 2021 / Accepted: 31 January 2022 / Published online: 1 March 2022

(c) The Author(s), under exclusive licence to Springer-Verlag GmbH Austria, part of Springer Nature 2022

\begin{abstract}
A novel electrochemical sensor is reported for the detection of the antiviral drug favipiravir based on the core-shell nanocomposite of flower-like molybdenum disulfide $\left(\mathrm{MoS}_{2}\right)$ nanospheres and molecularly imprinted polymers (MIPs). The $\mathrm{MoS}_{2} @$ MIP core-shell nanocomposite was prepared via the electrodeposition of a MIP layer on the $\mathrm{MoS}_{2}$ modified electrode, using o-phenylenediamine as the monomer and favipiravir as the template. The selective binding of target favipiravir at the $\mathrm{MoS}_{2} @$ MIP core-shell nanocomposite produced a redox signal in a concentration dependent manner, which was used for the quantitative analysis. The preparation process of the $\mathrm{MoS}_{2} @ \mathrm{MIP}$ core-shell nanocomposite was optimized. Under the optimal conditions, the sensor exhibited a wide linear response range of $0.01 \sim 100 \mathrm{nM}\left(1.57^{*} 10^{-6} \sim 1.57^{*} 10^{-2} \mu \mathrm{g} \mathrm{mL}^{-1}\right)$ and a low detection limit of $0.002 \mathrm{nM}\left(3.14 * 10^{-7} \mu \mathrm{g} \mathrm{mL} \mathrm{mL}^{-1}\right)$. Application of the sensor was demonstrated by detecting favipiravir in a minimum amount of $10 \mu \mathrm{L}$ biological samples (urine and plasma). Satisfied results in the recovery tests indicated a high potential of favipiravir monitoring in infectious COVID-19 samples.
\end{abstract}

Keywords Favipiravir $\cdot$ Electrochemical Sensor · Voltammetry, EIS $\cdot$ Molecularly imprinted polymer $\cdot$ Flower-like $\mathrm{MoS}_{2}$ nanospheres · Biological samples

\section{Introduction}

Favipiravir (FAV), a promising candidate of anti-coronavirus 2019 (COVID-19) drug, has been used in many countries around the globe [1]. The dosage regimen of FAV plays an important role in COVID-19 treatment, as inappropriate dosage may lead to subtherapeutic or toxic concentrations without clear clinical benefit [2]. Typically, the dosing recommendations are based on the pharmacokinetic investigation, which involves measuring drug concentrations in biological fluids at different time-points. However, the detection of FAV is considered to be a challenging job owing to the low target concentration and the high risk of infection in the samples collected from COVID-19 patients. Therefore, a suitable method to detect FAV is urgently required.

Weilu Liu

liuweilulucky@163.com

1 School of Pharmacy, Shenyang Pharmaceutical University, Shenyang 110016, People's Republic of China
Electrochemical sensor is becoming a promising technique for detecting drugs in biological fluids [3-6], not only because of its high sensitivity but also because of its rapid response and miniaturized instruments comparing with the traditional analytical techniques such as high-performance liquid chromatography (HPLC) $[7,8]$ and liquid chromatography-tandem mass spectrometry (LC-MS/MS) [9, 10]. Recently, an electrochemical sensor based on the Au @ Ag CSNPs/PEDOT:PSS/F-MWCNT composite has been reported for the detection of FAV in pharmaceutical and biological samples whereby wide linear range and low limit of detection were obtained owing to the synergistic effect of the electrode material [11]. Improved sensing performance were also obtained on the boron-doped diamond [12] and $\mathrm{MnO}_{2}$-rGO nanocomposite [13] modified electrodes, owing to the accumulation strategy used in the sensing process and the nanomaterial features including large surface area and good conductivity. These results demonstrated the feasibility of FAV detection using an electrochemical method.

The selectivity of the electrochemical sensors is another crucial factor that needs to be considered. Molecularly imprinted polymer (MIP), a biomimetic receptor with 
specific binding sites that are complementary in shape and size to target molecules, is becoming promising recognition element of electrochemical sensors. Compared with the biological counterpart such as antibodies and enzymes, the MIP receptor exhibits advantages of long-term stability, resistance to harsh environmental conditions, and low cost [14]. These features improve the reliability of the MIP sensor in the application of biological samples. Drugs and biomolecules have been used as the template of the MIPs, and satisfied selectivity was obtained based on the as-prepared sensors [15-18]. Electro-polymerization is a promising route to synthesize MIPs on the electrode surface whereby the thickness, topography, and morphology can be specifically manipulated [19]. Our group has developed several core-shell MIP nanocomposites by electrodepositing MIPs on the surface of carbon nanomaterials [20,21] and metal nanoparticles [22]. The as-prepared MIP layer exhibited large surface area and abundant binding sites, which improved the selectivity of the sensor. The nano-electrocatalyst, acting as the core-material to support the MIP layer, enhanced the sensitivity of the sensor by amplifying the redox signal. Considering the above aspects, it is promising to construct high performance electrochemical sensors by developing novel core-shell MIP nanocomposites.

Molybdenum disulfide $\left(\mathrm{MoS}_{2}\right)$, one of the transitionmetal dichalcogenides (TMDs), has attracted much attention as electrode material for sensing applications [23]. Owing to a unique sandwiched S-Mo-S layer structure stacked by weak van der Waals interaction, $\mathrm{MoS}_{2}$ exhibited high surface-to-volume ratio, high electron mobility, and abundant reactive sites for redox reactions. These features made $\mathrm{MoS}_{2}$ a promising supporting material for electrochemical sensors [24-27]. However, there were only a few reports of MIP sensors based on $\mathrm{MoS}_{2}$ nanomaterials [28-30].

In this work, a novel electrochemical sensor was developed based on $\mathrm{MoS}_{2} @$ MIP core-shell nanocomposite for the detection of anti COVID-19 drug of FAV. The flowerlike $\mathrm{MoS}_{2}$ nanospheres, prepared via a hydrothermal method, were used as the supporting material for MIP. A FAV imprinted MIP layer was then grafted on the surface of flower-like $\mathrm{MoS}_{2}$ nanospheres via an electrodeposition method. The selective FAV recognition and sensitive signal transduction were ensured by the unique $\mathrm{MoS}_{2} @ \mathrm{MIP}$ core-shell structure. Several parameters, including monomer/template ratio, electro-polymerization cycles, template elution time, and incubation time, have been carefully optimized to improve the sensing performance. Application of the sensor was investigated by detecting FAV in urine and plasma samples. Considering the specialty of the anti COVID-19 drug of FAV, a sample treatment method that required only $10 \mu \mathrm{L}$ of the biological samples was developed, and the accuracy of the sensor was evaluated by the recovery tests.

\section{Experimental}

\section{Materials and apparatus}

Favipiravir (FAV, 99\%) was purchased from Chenghui Shuangda Chemical Co., Ltd (Shandong, China). Dopamine hydrochloride $(\sim 98 \%)$, uric acid $(\sim 99 \%)$, o-phenylenediamine (o-PD, 98\%), and 5-fluorouracil ( 99\%) were obtained from Aladdin reagent China. Sodium molybdate dihydrate $\left(\mathrm{Na}_{2} \mathrm{MoO}_{4} \cdot 2 \mathrm{H}_{2} \mathrm{O}, \sim 99 \%\right)$, thioacetamide $\left(\mathrm{CH}_{3} \mathrm{CSNH}_{2}, \sim 99 \%\right)$, pyrazinamide $(\sim 98 \%)$, and 3-hydroxypyrazine-2-carboxamide ( $98 \%)$ were bought from Energy Chemical. Ascorbic acid (>99.7\%) and the other reagents were from Sinopharm chemical reagent.

Cyclic voltammetry (CV), differential pulse voltammetry (DPV), and electrochemical impedance spectroscopy (EIS) were performed on CHI660e workstation equipped with a three-electrode system, using a glassy carbon electrode (GCE, $3 \mathrm{~mm}$ diameter) as the working electrode, a $\mathrm{Pt}$ wire electrode as the counter electrode, and an $\mathrm{Ag} / \mathrm{AgCl}$ electrode as the reference electrode. The electrode materials were characterized by scanning electron microscopy (SEM, Hitachi-S4800) equipped with energy dispersive spectrometer (EDS), and transmission electron microscopy (TEM, JEM-2100).

\section{Synthesis of flower-like $\mathrm{MoS}_{2}$ nanospheres}

Flower-like $\mathrm{MoS}_{2}$ nanospheres were synthesized using a hydrothermal method [31]. The detailed procedure was provided in the Electronic Supporting Material.

\section{Preparation of $\mathrm{MoS}_{2} @ M I P$ core-shell nanocomposite}

The preparation of $\mathrm{MoS}_{2} @$ MIP core-shell nanocomposite was presented in Scheme 1. Firstly, a suspension of $\mathrm{MoS}_{2}(7 \mu \mathrm{L}, 0.1 \mathrm{mg} / \mathrm{mL}$ in DMF) was dropped onto the cleaned GCE surface and was left to dry at room temperature. Secondly, a MIP layer was electropolymerized on the $\mathrm{MoS}_{2}$-modified GCE by potential sweeping in the range of $0 \sim+0.8 \mathrm{~V}$ at a scan rate of $50 \mathrm{mV} / \mathrm{s}$ in $0.1 \mathrm{M}$ acetate buffer solution (ABS, pH 5.0) containing $10 \mathrm{mM}$ o-PD as monomer and $2 \mathrm{mM}$ FAV as template. The cyclic voltammogram in Fig. S1 exhibited an anodic peak at $0.4 \mathrm{~V}$ that can be attributed to the oxidation o-PD [32]. The peak current decreased under consecutive cyclic scans, indicating the insulating polymer film was formed on the $\mathrm{MoS}_{2}$-modified GCE. Thirdly, the template FAV was removed from the MIP by washing in an ethanol solution for $10 \mathrm{~min}$ at ambient temperature. Fourthly, the 
Scheme 1 Preparation of $\mathrm{MoS}_{2} @$ MIP core-shell nanocomposite

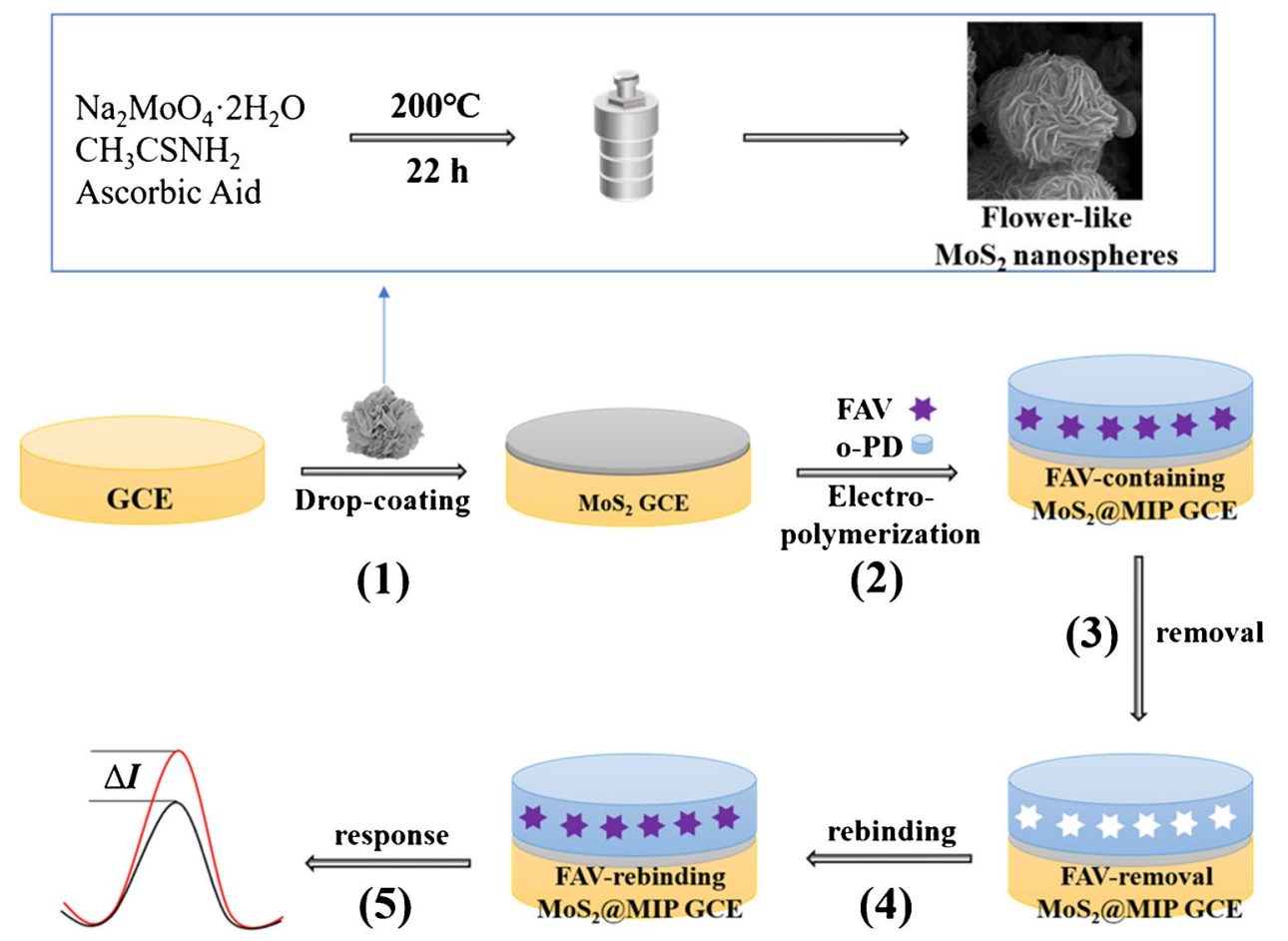

template-removed $\mathrm{MoS}_{2} @ \mathrm{MIP}$ core-shell nanocomposite was used to rebind and detect the target favipiravir. As control experiments, the $\mathrm{MoS}_{2} @ \mathrm{NIP}$-modified GCE was prepared in the same way but in the absence of favipiravir, and the MIP-modified GCE was also prepared in the absence of $\mathrm{MoS}_{2}$ nanospheres.

\section{Sample preparation and electrochemical tests}

Human urine $(10 \mu \mathrm{L})$ and Sprague Dawley rat plasma (10 $\mu \mathrm{L})$ samples were treated by mixing with $200 \mu \mathrm{L}$ of acetonitrile, respectively. The treated samples were centrifuged for $10 \mathrm{~min}$ at $11,000 \mathrm{rpm}$ and the supernatants were filtered and dried in $\mathrm{N}_{2}$. The residues were dissolved and diluted with ABS $(\mathrm{pH}=5.0)$ to a volume of $5 \mathrm{~mL}$. Then, $100 \mu \mathrm{L}$ of the solution was added into an electrolytic cell (Fig. S2) for testing.

The electrochemical tests were accomplished by recording the changes of DPV response of the redox probe $(5 \mathrm{mM}$ $\mathrm{K}_{3} \mathrm{Fe}(\mathrm{CN})_{6} / \mathrm{K}_{4}\left[\mathrm{Fe}(\mathrm{CN})_{6}\right]$ in $0.1 \mathrm{M} \mathrm{KCl}$ solution) at the $\mathrm{MoS}_{2} @$ MIP-modified electrode. Firstly, the modified electrode was immersed into a blank ABS (0.1 M, pH 5.0) for $5 \mathrm{~min}$, and the DPV response of the redox probe was recorded as $I_{0}$. Secondly, the modified electrode was incubated with the sample containing a certain concentration of FAV for $5 \mathrm{~min}$, and the DPV response of the redox probe was recorded as $I$. The decrease of the DPV peak current $\left(\Delta I=I_{0}-I\right)$, as illustrated in step (5) of Scheme 1, was used to evaluate the concentration of FAV in the samples.

\section{Results and discussion}

\section{Design of $\mathrm{MoS}_{2} @ M I P$ core-shell nanocomposite}

The unique $\mathrm{MoS}_{2} @ \mathrm{MIP}$ core-shell nanocomposite was designed for the sensitive and selective detection of FAV. The detection mechanism can be described using the gate effect at the MIP shell [33], whereby the FAV recognition signal was transferred into the inhibition of the electrochemical signal of the redox probe in a concentration dependent manner. The flower-like $\mathrm{MoS}_{2}$ nanospheres were chosen as the core material to support the MIP shell due to the unique merits. Firstly, the flower-like $\mathrm{MoS}_{2}$ nanospheres exhibited excellent electrochemical activity and enlarged surface area, which enhanced the electrochemical signal of the redox probe and improved the sensitivity of the sensor. Secondly, the loading of MIP shell on the flower-like core-material prevented the agglomeration of MIP matrix, and resulted in the abundant binding sites on surface of the sensor. Consequently, the recognition of the target favipiravir can be conducted in a more efficient way, which benefited both the selectivity and sensitivity. Therefore, the $\mathrm{MoS}_{2} @ \mathrm{MIP}$ core-shell nanocomposite was used as the sensing material for the detection of FAV.

\section{Characterization of $\mathrm{MoS}_{2} @ M I P$ core-shell nanocomposite}

The morphology of the $\mathrm{MoS}_{2} @ \mathrm{MIP}$ core-shell nanocomposite was characterized using SEM and TEM methods. The 
SEM images in Fig. 1A and B exhibited a flower-like pattern of $\mathrm{MoS}_{2}$ with numerous interlaced nanosheets as petals. The diameter of the $\mathrm{MoS}_{2}$ nanospheres and the thickness of the petals were measured around $394 \mathrm{~nm}$ and $10 \mathrm{~nm}$ (Fig. S3A and S3B). TEM images in Fig. 1C and D further demonstrated the microstructure of the flower-like $\mathrm{MoS}_{2}$ with interlaced, curved, and ultrathin nanosheets as petals. The elementary composition of the $\mathrm{MoS}_{2}$ was demonstrated by EDS analysis as shown in Fig. S3C. After electrodepositing the MIP shell, the electrode surface morphology was presented by the SEM images in Fig. 1E and F. It is observed that the flower-like $\mathrm{MoS}_{2}$ was evenly covered by the MIP shell, presenting a corrugated surface.

The electro-active surface areas of the modified electrodes were estimated by performing the cyclic voltammetry in $5 \mathrm{mM}$ $\mathrm{K}_{3} \mathrm{Fe}(\mathrm{CN})_{6} / \mathrm{K}_{4} \mathrm{Fe}(\mathrm{CN})_{6}$ (as shown in Fig.S4) and analyzed using the Randles-Sevcik equation [34].

$I_{\mathrm{p}}=2.69 \times 10^{5} A n^{3 / 2} D_{0}^{1 / 2} v^{1 / 2} C_{0}$ where $n$ is the number of electron transferred, $I_{\mathrm{p}}$ is the anodic peak current, $A$ is the effective surface area, $D_{0}$ is the diffusion coefficient, $v$ is the scan rate, and $C_{0}$ is the concentration.

The electro-active surface areas of MIP-modified electrode and $\mathrm{MoS}_{2} @ \mathrm{MIP}$ core-shell nanocomposite-modified electrode were calculated to be 0.34 and $0.81 \mathrm{~cm}^{2}$, respectively. It is demonstrated that using the flower-like $\mathrm{MoS}_{2}$ nanospheres as core material to support the MIP shell could increase the electrode effective surface area. Moreover, the heterogeneous rate constants $\left(k_{s}\right)$ at MIP-modified electrode and $\mathrm{MoS}_{2} @ \mathrm{MIP}$ core-shell nanocomposite-modified electrode were calculated as $0.3 \mathrm{~s}^{-1}$ and $0.6 \mathrm{~s}^{-1}$ according to the following equation [35].

$\log k_{s}=\alpha \log (1-\alpha)+(1-\alpha) \log \alpha-\log \frac{R T}{n F v}-\alpha(1-\alpha) \frac{n F \Delta E_{p}}{2.3 R T}$

where $\Delta E_{p}=E_{p a}-E_{p c}, v$ is the scan rate, and all other symbols have their conventional meanings.
Fig. 1 SEM images (A, B) and TEM images $(\mathbf{C}, \mathbf{D})$ of flower-like $\mathrm{MoS}_{2}$ nanospheres. SEM images of $\mathrm{MoS}_{2} @$ MIP core-shell nanocomposite on electrode surface $(\mathbf{E}, \mathbf{F})$
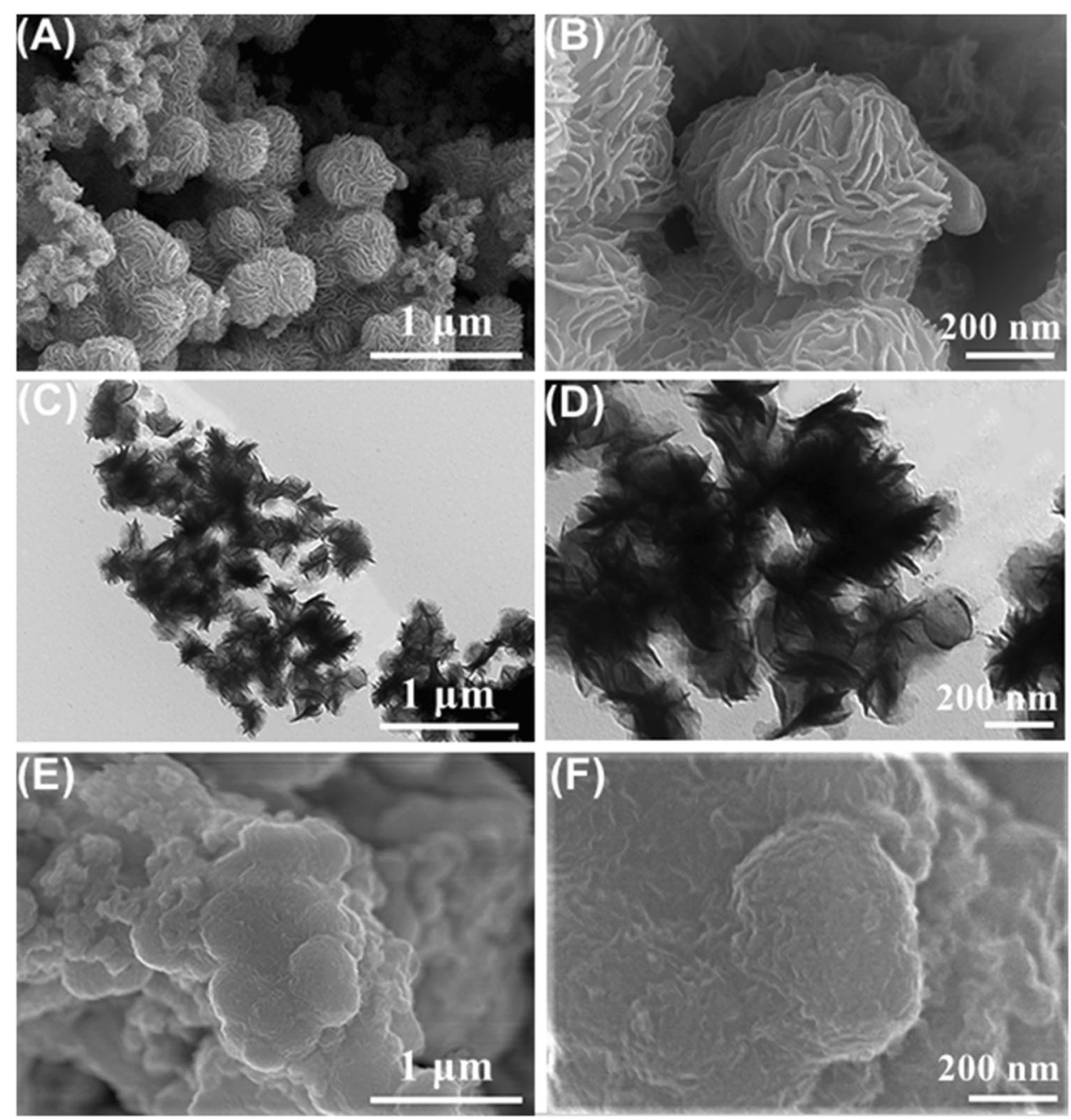


\section{Binding of target FAV}

The selective binding of target FAV on the $\mathrm{MoS}_{2} @ \mathrm{MIP}$ core-shell nanocomposite was evaluated using the theoretical simulation method. Firstly, GROMACS [36] was used to perform the molecular dynamic simulation study for establishing the stable complex between the template and monomer. The optimized structure as shown in Fig. 2A represented the FAV-imprinted binding site in the MIP polymer. The specific hydrogen-bonding and $\pi-\pi$ stacking between the monomer (o-PD) and the template (FAV) are responsible for the selective binding behavior. Secondly, AUTODOCK Vina was used to calculate the binding energy between the simulated binding site and the target FAV. The binding energy of the FAV-imprinted binding site was calculated as $-4.8 \mathrm{kcal} /$ mol (Table S1) using AUTODOCK Vina software package [37], which indicated a high binding ability of the MIP shell to the target FAV. More details about the GROMACS and AUTODOCK Vina calculations can be found in Electronic Supporting Material.

$\mathrm{CV}$ and EIS methods were used to further evaluate the binding ability of the $\mathrm{MoS}_{2} @ \mathrm{MIP}$ core-shell nanocomposite via the redox probe of $5 \mathrm{mM} \mathrm{K}{ }_{3} \mathrm{Fe}(\mathrm{CN})_{6} / \mathrm{K}_{4} \mathrm{Fe}(\mathrm{CN})_{6}$. $\mathrm{CV}$ analysis indicated the FAV-containing $\mathrm{MoS}_{2} @ \mathrm{MIP}$ core-shell-coated electrode exhibited barely response (curve-a in Fig. 2B), because the permeation of the redox probe was hindered. After the template FAV was removed, the binding sites in the MIP matrix allowed the redox probe to reach the electrode surface, resulting in the recovery in the $\mathrm{CV}$ response (curve-b in Fig. 2B). The subsequent rebinding of target FAV led to a decreased $\mathrm{CV}$ response (curve-c in Fig. 2B), owing to the binding sites were partially blocked by the rebinded FAV. The EIS spectrum in Fig. $2 \mathrm{C}$ further demonstrated the result of $\mathrm{CV}$ analysis. The charge transfer resistance (Rct) value, being calculated from the diameter of the semicircle, is considered to be inversely proportional to the electrochemical activity of the surface [38]. An extremely large Rct value in the EIS spectrum (curve-a in Fig. 2C) that indicates a blocked access from the electrode to the solution was found on the FAV-containing $\mathrm{MoS}_{2} @ \mathrm{MIP}$ core-shell nanocomposite-coated electrode. A decreased Rct value (curve-b in Fig. 2C) was observed after the template removal, and a re-increased Rct value (curve-c in Fig. 2C) was presented after the subsequent binding of the FAV. Both the CV and EIS analysis demonstrated that the binding of the target FAV could produce an obvious change of the redox signal, which could be used for the quantitative analysis.

\section{Optimization of the MoS $\mathrm{S}_{2} \mathrm{MIP}$ core-shell sensor}

The preparation of the $\mathrm{MoS}_{2} @ \mathrm{MIP}$ core-shell nanocomposite and sensing procedure of sensor have been investigated. The optimization experiments indicated that a better sensing performance can be obtained at the monomer/template ratio of 5:1, the electro-polymerization cycles of 20 , the template elution time of $10 \mathrm{~min}$, and the incubation time of $5 \mathrm{~min}$. The detailed discussions were provided in Fig. S5 in the Electronic Supporting Material. The detection of FAV was then conducted under the optimized conditions.
Fig. 2 A The simulated FAVimprinted binding sites obtained using GROMACS software. Hydrogen bonding and $\pi-\pi$ stacking are shown with yellow and black dashed lines, respectively. Carbon atoms are shown in green, hydrogen atoms in white, oxygen atoms in red, and nitrogen atoms in blue color. CV (B) and EIS $(\mathbf{C})$ performance of $5 \mathrm{mM} \mathrm{K}_{3} \mathrm{Fe}(\mathrm{CN})_{6} / \mathrm{K}_{4} \mathrm{Fe}(\mathrm{CN})_{6}$ obtained at $\mathrm{MoS}_{2} @ \mathrm{MIP}$ GCE before template removal (curve a), at $\mathrm{MoS}_{2} @$ MIP GCE after template removal (curve b), and at $\mathrm{MoS}_{2} @$ MIP GCE after rebinding of $10 \mathrm{nM}$ FAV (curve c). $\mathrm{CV}$ scan rate: $100 \mathrm{mV}^{-1}$
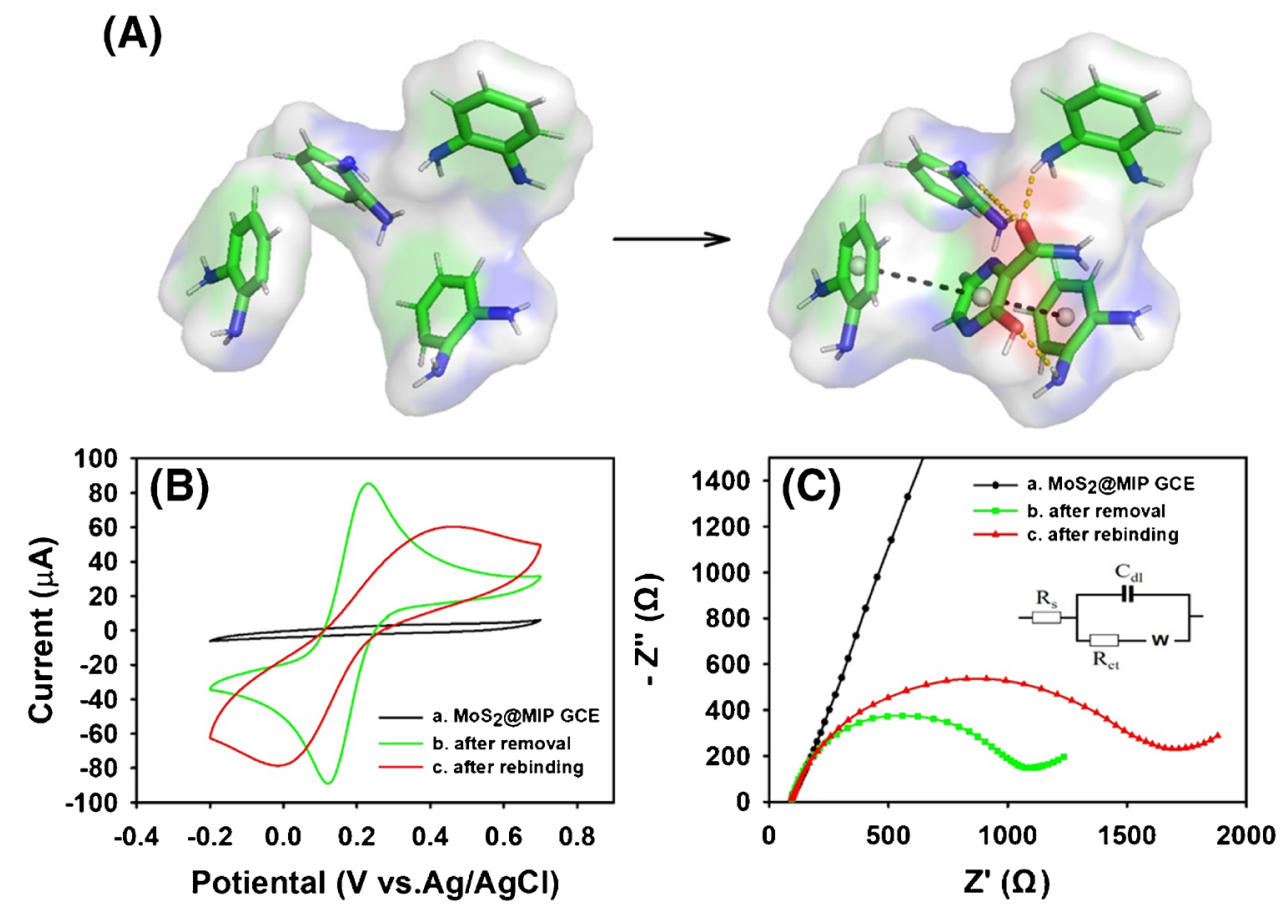


\section{Calibration curve and limit of detection}

Figure 3A exhibited the DPV responses of the redox probe obtained after incubation the electrodes with analyte FAV in different concentrations. At the $\mathrm{MoS}_{2} @$ MIP-modified electrode, the DPV peak currents decreased significantly with the increasing FAV concentrations in the concentration range of 0.01 to $100 \mathrm{nM}\left(1.57 * 10^{-6} \sim 1.57 * 10^{-2} \mu \mathrm{g} \mathrm{mL}{ }^{-1}\right)$, which can be attributed to the gate effect of MIP shell. The recognized FAV on the MIP shell blocked the access of redox probe to the electrode, and consequently resulted in a decreased DPV response comparing with that before FAV incubation. The linear relationship of the decrease of the DPV peak current $(\Delta I)$ versus the logarithm of FAV concentrations (Fig. 3B) indicated $\Delta I(\mu \mathrm{A})=10.97 \log c(\mathrm{nM})+38.54\left(\mathrm{R}^{2}=0.9767\right)$, and the limit of detection (LOD) was calculated to be $0.002 \mathrm{nM}(\mathrm{S} / \mathrm{N}=3)$.

At the MIP-modified electrode (prepared without $\mathrm{MoS}_{2}$ nanospheres), the DPV responses were obviously lower than that at the $\mathrm{MoS}_{2} @$ MIP-modified electrode, and the linear regression equation was expressed as $\Delta I(\mu \mathrm{A})=1.983 \log c(\mathrm{nM})+9.811\left(\mathrm{R}^{2}=0.9789\right)$. The enhanced sensitivity at the $\mathrm{MoS}_{2} @$ MIP sensor can be ascribed to the unique core-shell nanostructure. The flowerlike $\mathrm{MoS}_{2}$ core material increased the surface area of MIP shell and brought in a large number of binding sites, which benefited the recognition efficiency of the sensor. Moreover, the good conductivity and catalytic activity of the flowerlike $\mathrm{MoS}_{2}$ nanospheres could enhance the redox signal of the sensor.
At the $\mathrm{MoS}_{2} @$ NIP-modified electrode (prepared without template FAV), no FAV-specific binding sites were expected to recognize the target FAV. The slight DPV responses, deriving from non-specific adsorption of FAV on the NIP shell, were observed. The linear regression equation was expressed as $\Delta I(\mu \mathrm{A})=2.732 \log c(\mathrm{nM})+10.78\left(\mathrm{R}^{2}=0.9814\right)$. According to the sensitivity of $\mathrm{MoS}_{2} @ \mathrm{MIP}$ versus $\mathrm{MoS}_{2} @ \mathrm{NIP}$, the imprinting factor (IF) was calculated to be 4.6, indicating the good binding ability of the MIP shell.

Table 1 exhibited the comparison of the proposed FAV detection method with some reported methods including both the chromatographic methods and electrochemical methods. The as-prepared $\mathrm{MoS}_{2} @$ MIP sensor exhibited relatively wider linear range, and the lowest LOD of $0.002 \mathrm{nM}$ that was at least two orders of magnitudes lower than the previously reported methods. The excellent analytical performance can be attributed to the abundant binding sites and high electrochemical activity at the $\mathrm{MoS}_{2} @$ MIP core-shell nanocomposite. The as-prepared $\mathrm{MoS}_{2} @$ MIP sensor has a different sensing mechanism comparing with previously reported FAV electrochemical sensors. The $\mathrm{MoS}_{2} @ \mathrm{MIP}$ sensor works by transferring the FAV recognition signal at the MIP shell into a sensitive electrochemical signal of the redox probe, instead of direct oxidizing FAV at the electrode surface under a high potential, which consequently prevented the interference of some electroactive compounds in biological matrix and improved the sensing accuracy. However, the as-prepared $\mathrm{MoS}_{2} @$ MIP sensor required two separated steps for FAV sensing including the incubation of the electrode in the target FAV solution and the following
Fig. 3 A DPV responses of $5 \mathrm{mM} \mathrm{K}_{3} \mathrm{Fe}(\mathrm{CN})_{6} / \mathrm{K}_{4} \mathrm{Fe}(\mathrm{CN})_{6}$ at the different modified electrodes after incubation with FAV of different concentrations (from top to bottom: blank ABS, $0.01,0.1,1,10,100 \mathrm{nM}) . \mathbf{B}$ The calibration plot of the $\Delta I$ values (the decrease of DPV peak current after incubation with FAV) versus the logarithm of FAV concentration. C The chemical structures of (1) favipiravir, (2) pyrazinamide, (3) 3-hydroxypyrazine-2-carboxamide, (4) 5-fluorouracil, (5) uric acid, (6) ascorbic acid, and (7) dopamine. D The change of the current response of $0.1 \mathrm{nM}$ FAV after the addition of the interfering substances $(n=3)$ at $\mathrm{MoS}_{2} @$ MIP nanocomposite
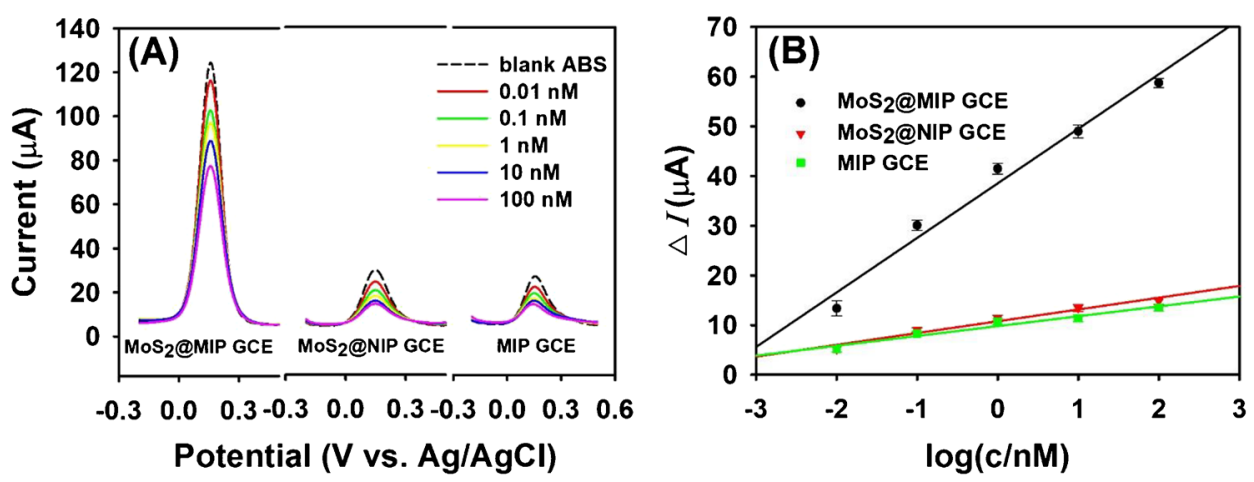

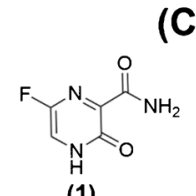

(1)

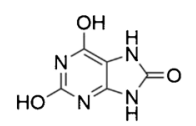

(5)

(C)

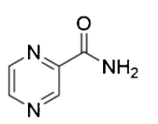

(2)

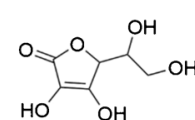

(6)

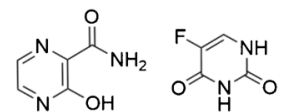

(3)

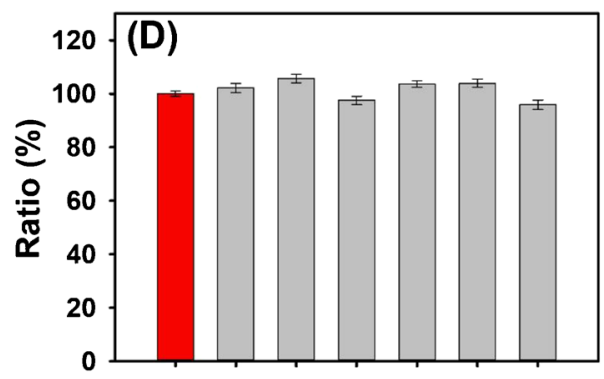

(1) (2) (3) (4) (5) (6) (7) 
Table 1 Comparison of methods used in determination of FAV

\begin{tabular}{|c|c|c|c|c|}
\hline Method & Electrode materials & Linear range $(\mu \mathrm{M})$ & $\mathrm{LOD}(\mu \mathrm{M})$ & Ref \\
\hline HPLC & - & $63.6 \sim 636.5$ & 7.6 & [8] \\
\hline LC-MS/MS & - & $0.63 \sim 127.3$ & 0.15 & [10] \\
\hline DPV & $\begin{array}{c}\text { Au @ Ag CSNPs/ } \\
\text { PEDOT:PSS/F- } \\
\text { MWCNT GCE }^{\text {a }}\end{array}$ & $0.005 \sim 2$ & 0.0046 & [11] \\
\hline$S W V^{b}$ & CPT-BDD GCE ${ }^{\mathrm{c}}$ & $0.064 \sim 130$ & 0.018 & {$[12]$} \\
\hline SWV & $\mathrm{MnO}_{2}-\mathrm{rGO} \mathrm{SPE}^{\mathrm{d}}$ & $0.01 \sim 55$ & 0.009 & {$[13]$} \\
\hline DPV & $\mathrm{MoS}_{2} @$ MIP-GCE & $1.0^{*} 10^{-5} \sim 1.0^{*} 10^{-1}$ & $2 * 10^{-6}$ & This work \\
\hline
\end{tabular}

${ }^{a}$ Gold/silver core-shell nanoparticles with conductive polymer poly (3,4-ethylenedioxythiophene) polystyrene sulfonate and functionalized multi carbon nanotubes glassy carbon electrodes

${ }^{\mathrm{b}}$ Square-wave voltammetry

${ }^{\mathrm{c}}$ Cathodically pretreated boron-doped diamond glassy carbon electrodes

${ }^{\mathrm{d}} \mathrm{MnO}_{2}$-reduced graphene oxide screen-printing electrodes

electrochemical detection of the redox probe signal. This two-step procedure may not be as facile as that of the onestep direct electrochemical oxidation method. Considering this aspect, further efforts can be made to anchor the redox probe on the electrode surface, which may open the possibility of the direct detection of the MIP recognition signal in a single step.

\section{Selectivity, reproducibility, stability, and reusability}

The selectivity of the $\mathrm{MoS}_{2} @$ MIP electrode was evaluated by detecting FAV in the presence of structurally similar compounds (pyrazinamide, 3-hydroxypyrazine-2-carboxamide, and 5-fluorouracil) and potential interference compounds (uric acid, ascorbic acid, and dopamine). The chemical structures of the analytes are shown in Fig. 3C. The current response at the $\mathrm{MoS}_{2} @$ MIP electrode maintained almost stable after the addition of the other compounds. The change of the current response was found to be between 95.9 and $105.7 \%$ (Fig. 3D), indicating the high selectivity of FAV detection at the $\mathrm{MoS}_{2} @ \mathrm{MIP}$ sensor.

The reproducibility was evaluated by measuring $0.1 \mathrm{nM}$ FAV with five sensors prepared under the same condition. The relative standard deviation (RSD) of the obtained results was $4.1 \%$, indicating the satisfying reproducibility of the sensor preparing method. The stability was investigated by measuring $0.1 \mathrm{nM}$ FAV after the sensor was stored for 14 days. It showed that the sensor still presented $90.6 \%$ of its initial current response. The good reproducibility and stability of the sensor may derive from the chemical and mechanical stability of the $\mathrm{MoS}_{2} @$ MIP core shell structure. The reusability was evaluated by measuring $0.1 \mathrm{nM}$ FAV at one $\mathrm{MoS}_{2} @$ MIP sensor for 5 times. After each detection, the $\mathrm{MoS}_{2} @$ MIP electrode was washed with $5 \mathrm{~mL}$ ethanol for $5 \mathrm{~min}$ to remove the adsorbed FAV. The
RSD of the 5 detection results was $6.8 \%$, indicating the acceptable reusability of the $\mathrm{MoS}_{2} @$ MIP sensor.

\section{Real sample analysis}

For investigating the accuracy of the fabricated $\mathrm{MoS}_{2} @$ MIP sensor, the sensor was applied to detect FAV in human urine and rat plasma samples. Recovery tests were applied to demonstrate the accuracy of the as-prepared sensor by using the following formula:

Recovery $(\%)=\frac{\text { Found }}{\text { Added }} \times 100 \%$

The results in Table 2 exhibited good accuracy with recoveries ranging from 86.6 to $119.0 \%$ in urine sample, and from 81.6 to $103.6 \%$ in plasma sample. These results demonstrated the reliability in the application of biological samples.

Table 2 Detection of FAV in urine and plasma samples $(n=3)$

\begin{tabular}{lllll}
\hline Samples & Added $(\mathrm{nM})$ & Found $(\mathrm{nM})$ & Recovery $(\%)$ & RSD $(\%)$ \\
\hline Urine & 0.05 & $0.050 \pm 0.0007$ & 100.0 & 1.4 \\
& 0.5 & $0.595 \pm 0.006$ & 119.0 & 1.0 \\
& 5 & $4.331 \pm 0.09$ & 86.6 & 2.0 \\
Plasma & 0.05 & $0.042 \pm 0.001$ & 84.0 & 2.3 \\
& 0.5 & $0.518 \pm 0.01$ & 103.6 & 2.5 \\
& 5 & $4.080 \pm 0.1$ & 81.6 & 3.6 \\
\hline
\end{tabular}




\section{Conclusion}

In this work, a novel $\mathrm{MoS}_{2} @ \mathrm{MIP}$ nanocomposite was developed and characterized using SEM, TEM, EDS, CV, and EIS. According to the characterization studies, the asprepared nanocomposite exhibited a core-shell structure of flower-like $\mathrm{MoS}_{2}$ nanosphere (core) and MIP (shell). A sensing mechanism of transferring the recognition signal at the MIP shell to a sensitive electrochemical signal of the redox probe was demonstrated for the selective and sensitive detection of FAV in biological samples including human urine and rat plasma. The as-prepared sensor also exhibited high reproducibility, stability, reusability, and recovery in the sensing application. The improved analytical performance can be attributed to the abundant binding sites and high electrochemical activity at the $\mathrm{MoS}_{2} @ \mathrm{MIP}$ core-shell nanocomposite. It is concluded that the novel $\mathrm{MoS}_{2} @ \mathrm{MIP}$ nanocomposite can be used as a promising sensing material for the detection of FAV in real samples.

Supplementary Information The online version contains supplementary material available at https://doi.org/10.1007/s00604-022-05213-9.

Funding This work was supported by the National Natural Science Foundation of China (No. 81503037), Natural Science Foundation of Liaoning Province (2021-MS-211), Career Development Support Plan for Young and Middle-aged Teachers in Shenyang Pharmaceutical University (No. ZQN2015029), and Basic research projects of Liaoning Provincial Department of Education (No. 2017LQN06).

\section{Declarations}

Conflict of interest The authors declare no competing interests.

\section{References}

1. Agrawal U, Raju R, Udwadia ZF (2020) Favipiravir: a new and emerging antiviral option in COVID-19. Medical journal, Armed Forces India 76:370-376. https://doi.org/10.1016/j.mjafi.2020.08. 004

2. Habler K, Brugel M, Teupser D, Liebchen U, Scharf C, Schonermarck U, Vogeser M, Paal M (2021) Simultaneous quantification of seven repurposed COVID-19 drugs remdesivir (plus metabolite GS-441524), chloroquine, hydroxychloroquine, lopinavir, ritonavir, favipiravir and azithromycin by a two-dimensional isotope dilution LC-MS/MS method in human serum. J Pharm Biomed Anal 196:113935. https://doi.org/10.1016/j.jpba.2021.113935

3. Beluomini MA, da Silva JL, Sedenho GC, Stradiotto NR (2017) D-mannitol sensor based on molecularly imprinted polymer on electrode modified with reduced graphene oxide decorated with gold nanoparticles. Talanta 165:231-239. https://doi.org/10. 1016/j.talanta.2016.12.040

4. Maduraiveeran G, Sasidharan M, Ganesan V (2018) Electrochemical sensor and biosensor platforms based on advanced nanomaterials for biological and biomedical applications. Biosens Bioelectron 103:113-129. https://doi.org/10.1016/j.bios.2017.12.031

5. Mahmoud AM, El Wekil MM, Mahnashi MH, Ali MFB, Alkahtani SA (2019) Modification of N, S co-doped graphene quantum dots with p-aminothiophenol-functionalized gold nanoparticles for molecular imprint-based voltammetric determination of the antiviral drug sofosbuvir. Microchim Acta 186:617. https:// doi.org/10.1007/s00604-019-3647-7

6. Martinez-Perinan E, Garcia-Mendiola T, Enebral-Romero E, del Cano R, Vera-Hidalgo M, Sulleiro MV, Navio C, Pariente F, Perez EM, Lorenzo E (2021) A MoS2 platform and thionine-carbon nanodots for sensitive and selective detection of pathogens. Biosens Bioelectron 189:113375. https://doi.org/10.1016/j.bios.2021. 113375

7. Moriiwa Y, Morikawa G, Okazawa K, Yanagida A (2021) Optimization of analytical procedure for in-hospital rapid quantification of serum level of favipiravir in the pharmacological treatment of COVID-19. Anal Sci 37:1301-1304. https://doi.org/10.2116/anals ci.21N004

8. Bulduk I (2021) HPLC-UV method for quantification of favipiravir in pharmaceutical formulations. Acta Chromatogr 33:209-215. https://doi.org/10.1556/1326.2020.00828

9. Rezk MR, Badr KA, Abdel-Naby NS, Ayyad MM (2021) A novel, rapid and simple UPLC-MS/MS method for quantification of favipiravir in human plasma: application to a bioequivalence study. Biomed Chromatogr 35:e5098. https://doi.org/10.1002/bmc.5098

10. Morsy MI, Nouman EG, Abdallah YM, Zainelabdeen MA, Darwish MM, Hassan AY, Gouda AS, Rezk MR, Abdel-Megied AM, Marzouk HM (2021) A novel LC-MS/MS method for determination of the potential antiviral candidate favipiravir for the emergency treatment of SARS-CoV-2 virus in human plasma: Application to a bioequivalence study in Egyptian human volunteers. J Pharm Biomed Anal 199. https://doi.org/10.1016/j.jpba.2021. 114057

11. Mehmandoust M, Khoshnavaz Y, Tuzen M, Erk N (2021) Voltammetric sensor based on bimetallic nanocomposite for determination of favipiravir as an antiviral drug. Microchim Acta 188. https://doi.org/10.1007/s00604-021-05107-2

12. Allahverdiyeva S, Yunusoglu O, Yardim Y, Senturk Z (2021) First electrochemical evaluation of favipiravir used as an antiviral option in the treatment of COVID-19: a study of its enhanced voltammetric determination in cationic surfactant media using a boron-doped diamond electrode. Anal Chim Acta 1159:338418. https://doi.org/10.1016/j.aca.2021.338418

13. Mohamed MA, Eldin GMG, Ismail SM, Zine N, Elaissari A, Jaffrezic-Renault N, Errachid A (2021) Innovative electrochemical sensor for the precise determination of the new antiviral COVID19 treatment Favipiravir in the presence of coadministered drugs. J Electroanal Chem 895:115422-115422. https://doi.org/10.1016/j. jelechem.2021.115422

14. Cieplak M, Kutner W (2016) Artificial biosensors: how can molecular imprinting mimic biorecognition? Trends Biotechnol 34:922-941. https://doi.org/10.1016/j.tibtech.2016.05.011

15. Stoian IA, Iacob BC, Dudas CL, Barbu-Tudoran L, Bogdan D, Marian IO, Bodoki E, Oprean R (2020) Biomimetic electrochemical sensor for the highly selective detection of azithromycin in biological samples. Biosens Bioelectron 155:112098. https://doi. org/10.1016/j.bios.2020.112098

16. Pardeshi S, Singh SK (2016) Precipitation polymerization: a versatile tool for preparing molecularly imprinted polymer beads for chromatography applications. RSC Adv 6:23525-23536. https:// doi.org/10.1039/c6ra02784a

17. Lian W, Liu S, Wang L, Liu H (2015) A novel strategy to improve the sensitivity of antibiotics determination based on bioelectrocatalysis at molecularly imprinted polymer film electrodes. Biosens Bioelectron 73:214-220. https://doi.org/10.1016/j.bios.2015.06. 006

18. Jyoti GC, Zolek T, Maciejewska D, Kutner A, Merlier F, Haupt K, Sharma PS, Noworyta KR, Kutner W (2021) Molecularly imprinted polymer nanoparticles-based electrochemical 
chemosensors for selective determination of cilostazol and its pharmacologically active primary metabolite in human plasma. Biosens Bioelectron 193:113542. https://doi.org/10.1016/j.bios. 2021.113542

19. Crapnell RD, Hudson A, Foster CW, Eersels K, van Grinsven B, Cleij TJ, Banks CE, Peeters M (2019) Recent advances in electrosynthesized molecularly imprinted polymer sensing platforms for bioanalyte detection. Sensors-Basel 19. https://doi.org/10. 3390/s19051204

20. Zhao XY, Liu YZ, Zuo JJ, Zhang J, Zhu L, Zhang JK (2017) Rapid and sensitive determination of tartrazine using a molecularly imprinted copolymer modified carbon electrode (MIP-PmDB/ PoPD-GCE). J Electroanal Chem 785:90-95. https://doi.org/10. 1016/j.jelechem.2016.12.015

21. Liu W, Cui F, Li H, Wang S, Zhuo B (2020) Three-dimensional hybrid networks of molecularly imprinted poly (9-carbazoleacetic acid) and MWCNTs for simultaneous voltammetric determination of dopamine and epinephrine in plasma sample. Sens Actuators, B 323:128669. https://doi.org/10.1016/j.snb.2020.128669

22. Zheng W, Xiong Z, Li H, Yu S, Li G, Niu L, Liu W (2018) Electrodeposited Pt@Molecularly imprinted polymer core-shell nanostructure: enhanced sensing platform for sensitive and selective detection of bisphenol A. Sens Actuators, B 272:655-661. https:// doi.org/10.1016/j.snb.2018.07.039

23. Sha R, Bhattacharyya TK (2020) MoS2-based nanosensors in biomedical and environmental monitoring applications. Electrochim Acta 349:136370. https://doi.org/10.1016/j.electacta.2020.136370

24. Mejri A, Mars A, Elfil H, Hamzaoui AH (2020) Curcumin graphite pencil electrode modified with molybdenum disulfide nanosheets decorated gold foams for simultaneous quantification of nitrite and hydrazine in water samples. Anal Chim Acta 1137:19-27. https://doi.org/10.1016/j.aca.2020.08.032

25. Zhang C, Ping J, Ye Z, Ying Y (2020) Two-dimensional nanocomposite-based electrochemical sensor for rapid determination of trans-resveratrol. Sci Total Environ 742:140351. https://doi. org/10.1016/j.scitotenv.2020.140351

26. Joswig J-O, Lorenz T, Wendumu TB, Gemming S, Seifert G (2015) Optics, Mechanics, and Energetics of Two-Dimensional MoS2 Nanostructures from a Theoretical Perspective. Acc Chem Res 48:48-55. https://doi.org/10.1021/ar500318p

27. Sha R, Vishnu N, Badhulika S (2019) MoS2 based ultra-low-cost, flexible, non-enzymatic and non-invasive electrochemical sensor for highly selective detection of Uric acid in human urine samples. Sens Actuators, B 279:53-60. https://doi.org/10.1016/j.snb.2018. 09.106

28. Li Y-T, Yang Y-Y, Sun Y-X, Cao Y, Huang Y-S, Han S (2020) Electrochemical fabrication of reduced MoS2-based portable molecular imprinting nanoprobe for selective SERS determination of theophylline. Microchim Acta 187:203. https://doi.org/10. 1007/s00604-020-4201-3
29. Wang Y, Zhang B, Tang Y, Zhao F, Zeng B (2021) Fabrication and application of a rutin electrochemical sensor based on rose-like AuNPs-MoS2-GN composite and molecularly imprinted chitosan. Microchem J 168:106505. https://doi.org/10.1016/j.microc.2021. 106505

30. Liang AA, Hou BH, Tang CS, Sun DL, Luo EA (2021) An advanced molecularly imprinted electrochemical sensor for the highly sensitive and selective detection and determination of Human IgG. Bioelectrochemistry 137:107671. https://doi.org/ 10.1016/j.bioelechem.2020.107671

31. Zheng XD, Zhu YL, Sun YL, Jiao QJ (2018) Hydrothermal synthesis of MoS2 with different morphology and its performance in thermal battery. J Power Sources 395:318-327. https://doi.org/10. 1016/j.jpowsour.2018.05.092

32. Yan C, Zhang R, Chen Y, Wang G (2017) Electrochemical determination of enrofloxacin based on molecularly imprinted polymer via one-step electro-copolymerization of pyrrole and o-phenylenediamine. J Electroanal Chem 806:130-135. https://doi.org/10. 1016/j.jelechem.2017.10.047

33. Wang D, Wang J, Zhang J, Li Y, Zhang Y, Li Y, Ye B-C (2019) Novel electrochemical sensing platform based on integration of molecularly imprinted polymer with Au@ Ag hollow nanoshell for determination of resveratrol. Talanta 196:479-485. https://doi.org/ 10.1016/j.talanta.2018.12.063

34. Bard AJ, Faulkner LR (1980) Electrochemical methods: fundamentals and applications. Wiley, New York. https://doi.org/10. 1021/ed060pa25.1

35. Laviron E (1979) General expression of the linear potential sweep voltammogram in the case of diffusionless electrochemical systems. J Electroanal Chem Interfacial Electrochem 101:19-28. https://doi.org/10.1016/S0022-0728(79)80075-3

36. Mark James Abraham TM, Schulz R, Páll S, Smith JC, Hess B, Lindahl E (2015) GROMACS: high performance molecular simulations through multi-level parallelism from laptops to supercomputers. SoftwareX 1-2:19-25. https://doi.org/10.1016/j.softx. 2015.06.001

37. Trott O, Olson AJ (2010) Software news and update AutoDock Vina: improving the speed and accuracy of docking with a new scoring function, efficient optimization, and multithreading. $\mathbf{J}$ Comput Chem 31:455-461. https://doi.org/10.1002/jcc.21334

38. Liu W, Li H, Yu S, Zhang J, Zheng W, Niu L, Li G (2018) Poly(3,6-diamino-9-ethylcarbazole) based molecularly imprinted polymer sensor for ultra-sensitive and selective detection of 17-beta-estradiol in biological fluids. Biosens Bioelectron 104:79_ 86. https://doi.org/10.1016/j.bios.2018.01.002

Publisher's Note Springer Nature remains neutral with regard to jurisdictional claims in published maps and institutional affiliations. 